SECCIÓN ESPECIAL

\title{
DURACIÓN DE LA GESTIÓN MINISTERIAL Y LOGROS EN SALUD EN EL PERÚ
}

\author{
Ernesto Gozzer(1,a, Fredy Canchihuamán(101,2,b, Vilma Chalco(1)1,c, Gabriela Huarii1,d, \\ Ana Cisneros-Dionisio(11,e, Wildo Quispe (1)1,c, Rubén Espinoza(1) 1,a \\ 1 Unidad de Seguridad Sanitaria Global y Diplomacia en Salud, Facultad de Salud Pública y Administración, Universidad \\ Peruana Cayetano Heredia, Lima, Perú. \\ 2 Programa de Líderes en Salud Internacional. Organización Panamericana de la Salud, Washington D.C., US. \\ ${ }^{a}$ Médico cirujano, MSc; ${ }^{\mathrm{b}}$ médico cirujano, $\mathrm{MPH}, \mathrm{PhD} ;{ }^{\mathrm{c}}$ enfermero; ${ }^{\mathrm{d}}$ cirujano dentista; ${ }^{\mathrm{e}}$ químico farmacéutico.
}

\section{RESUMEN}

Los periodos breves en la gestión de un ministerio de salud pueden poner en riesgo la implementación y la efectividad de políticas de Estado por cambios en la orientación de la gestión, por un proceso natural de aprendizaje, o por otros factores. El propósito de este artículo fue determinar y comparar la duración de la gestión ministerial en salud en Perú, describir sus características y discutir su relación con los logros en salud pública. Entre 1935 y 2021, la media de duración de la gestión fue de 13,7 meses y la mediana fue de 11 meses, siendo la más extensa de 67 meses, de Constantino Carvallo, y la más breve de un día, de Javier Correa. La duración de la gestión ministerial en el Perú tiene un amplio rango de variación (1 día hasta 67 meses), es menor comparada con la de otros países, y está disminuyendo desde el 2016. Si bien, una duración mayor a la encontrada no sería suficiente para garantizar los logros en salud, podría contribuir en el sostenimiento de las acciones de salud y tener un efecto positivo en las políticas de largo plazo.

Palabras claves: Agencias Gubernamentales; Administración en Salud Pública; Reorganización del Personal; Factores de Tiempo; Políticas Públicas de Salud; Salud Pública; Perú (Fuente: DeCS BIREME).

\section{LENGTH OF MINISTERIAL TENURE AND PUBLIC HEALTH ACHIEVEMENTS IN PERU ABSTRACT}

\begin{abstract}
Short administration periods of a health ministry can jeopardize the implementation and effectiveness of state policies due to changes in management orientation, altering the natural learning process, or other factors. The aim of this article was to determine and compare the tenure length of the ministry of health in Peru, describe its characteristics and discuss its relationship with public health achievements. Between 1935 and 2021, the average tenure was of 13,7 months and the median was 11 months, the longest was found to be 67 months, by Constantino Carvallo, and the shortest was one day, by Javier Correa. The length of ministerial tenure in Peru has a wide range of variation (1 day up to 67 months), it is shorter compared to that of other countries and is decreasing since 2016. Although longer tenures would not be sufficient to guarantee health achievements, it might contribute to the sustainability of health-related actions and have a positive effect on long-term policies.
\end{abstract}

Keywords: Government Agencies; Public Health Administration; Personnel Turnover; Time Factors; Public Health Policy; Public Health; Peru (Source: MeSH NLM).

F, Chalco V, Huari G, Cisneros-

Dionisio A, Quispe W, et al. Duración de la gestión ministerial y logros en salud en el Perú. Rev Peru Med Exp Salud Publica. 2021;38(2):32636. doi: https://doi.org/10.17843/ rpmesp.2021.382.6721.

Correspondencia: Fredy Antonio Canchihuamán Rivera; Av. Honorio Delgado 430, Urb. Ingeniería, San Martín de Porres; Perú. canchihuaman.r@upch.pe

\section{INTRODUCCIÓN}

Durante el siglo XIX los Estados empezaron a mostrar mayor interés por la protección de la salud de su población debido, entre otros factores, a los efectos que la salud podría tener sobre las relaciones entre los países ${ }^{(1)}$. La presencia formal y autónoma del sector salud se consolidó dentro de la estructura de los Estados con la creación del primer ministerio de salud en el mundo; que ocurrió en Cuba el 28 de enero de $1909^{(2)}$. En el Perú, 26 años más tarde (el 5 de octubre de 1935) se creó el Ministerio de Salud Pública, Trabajo y Previsión Social.

Con la globalización, los ministerios de salud progresivamente reconstituyeron su organización y dinamizaron su rol de acuerdo a la realidad de cada país y a la necesidad de asumir nuevas funciones, separarlas o delegarlas a organismos subnacionales $\mathrm{u}$ otros sectores ${ }^{(3,4)}$. El liderazgo en los ministerios de salud ha sido clave para impulsar, alcanzar y sostener logros 
en materia de salud e implementar complejos procesos de reforma sanitaria. No obstante, los logros sanitarios y los objetivos de una reforma podrían ponerse en riesgo si las políticas al respecto no trascienden a los eventuales cambios de liderazgo en los ministerios.

En la gestión pública el cargo más alto de un sector es el de ministro de Estado. Las designaciones ministeriales obedecen a reglas de juego resultantes de la situación política de los países. La permanencia en el cargo de un ministro puede depender, de igual forma, de la estabilidad del sistema político; estabilidad que por momentos es altamente dinámica. La frecuente rotación de los ministros de Estado - que se traduce usualmente en una alta rotación de directivos a su cargo- ocurre especialmente en los países en los que el servicio civil público no está institucionalizado y no responde a una designación necesariamente en base a méritos.

Diversos estudios han descrito una asociación positiva entre las diferentes características de una burocracia pública efectiva como la designación meritocrática y la estabilidad de los altos funcionarios y variables resultantes como el crecimiento económico, la reducción de la pobreza y el control de la corrupción ${ }^{(5-7)}$.

En el Perú, el ministro de Salud tiene la máxima responsabilidad política y ejecutiva del sector salud; sus facultades y funciones le permiten conducir el sistema nacional de salud; definir, monitorear y evaluar las políticas; articular con diferentes sectores; designar directivos; y aprobar los presupuestos ${ }^{(8)}$.

El propósito de este artículo fue determinar y comparar la duración de los cargos ministeriales específicos al sector salud y describir las características de los ministros designados desde la creación del Ministerio de Salud del Perú en 1935 hasta la actualidad. Un propósito adicional fue identificar los logros más significativos en salud pública durante este periodo.

\section{ABORDAJE METODOLÓGICO}

Se realizó un análisis descriptivo utilizando fuentes secundarias. Se revisó las normas legales del Diario Oficial El Peruano, donde se identificaron las designaciones a los cargos ministeriales y las correspondientes fechas; se revisó la base de datos del Registro Nacional de Identificación y Estado Civil (RENIEC) para obtener los nombres completos, fechas y lugares de nacimiento; la base de datos de médicos inscritos en el Colegio Médico del Perú y la información proporcionada por el Ministerio de Salud. Información adicional acerca de las características de los ministros fue obtenida de otras fuentes publicadas en revistas científicas, libros, Internet e información pública proporcionada por familiares, excolaboradores y en algunos casos los propios exministros.

Para la identificación de eventos importantes se revisó una serie de documentos que sintetizan la historia de salud pública desde el periodo próximo a la fundación del Ministerio de Salud hasta el $2021^{(9-16)}$. Se tomó en consideración las fechas de los eventos y como criterios de elección el efecto significativo de una estrategia, el impacto sobre la salud de la población o la transformación del sistema de salud. La información fue ingresada en una base de datos para su análisis.

\section{ANÁLISIS DESCRIPTIVO DE LAS GESTIONES MINISTERIALES}

Un total de 64 personas ocuparon el cargo de ministro desde la creación del Ministerio de Salud en 1935 hasta julio del 2021. Del total de funcionarios designados, la mayoría fue-

Tabla 1. Características sociodemográficas de los ministros de Salud del Perú durante su gestión en el cargo entre 1935 y 2021.

\begin{tabular}{|c|c|}
\hline Características & $\mathrm{N}=74(\%)$ \\
\hline \multicolumn{2}{|l|}{ Sexo } \\
\hline Femenino & $9(12)$ \\
\hline Masculino & $65(88)$ \\
\hline \multicolumn{2}{|l|}{ Edad al inicio de gestión (años) a } \\
\hline Menor de 40 & $4(6)$ \\
\hline 41 a 50 & $27(38)$ \\
\hline 51 a 60 & $30(42)$ \\
\hline Mayor de 60 & $10(14)$ \\
\hline \multicolumn{2}{|l|}{ Lugar de nacimiento $^{\text {a }}$} \\
\hline Lima & $39(56)$ \\
\hline Otra ciudad & $29(41)$ \\
\hline Ciudad del exterior & $2(3)$ \\
\hline \multicolumn{2}{|l|}{ Profesión } \\
\hline Médico & $54(73)$ \\
\hline Militar & $13(17)$ \\
\hline Economista & $2(3)$ \\
\hline Abogado & $2(3)$ \\
\hline Ingeniero & $2(3)$ \\
\hline Biólogo & $1(1)$ \\
\hline Especialidad médica a & $33(45)$ \\
\hline \multicolumn{2}{|l|}{ Posgrado $^{a}$} \\
\hline Maestría & $22(30)$ \\
\hline Doctorado & $17(23)$ \\
\hline \multicolumn{2}{|l|}{ Área de trabajo ${ }^{a}$} \\
\hline Clínica & $32(44)$ \\
\hline Salud pública, gestión u otras & $20(28)$ \\
\hline No relacionada a salud & $20(28)$ \\
\hline Participación política ${ }^{a}$ & $31(42)$ \\
\hline
\end{tabular}


ron hombres y procedentes de Lima (Tabla 1). Nueve fueron mujeres (12\%); la primera ministra de Estado en el Perú y de la cartera de Salud fue Ilda Urizar, quien estuvo en el cargo entre junio de 1987 y mayo de 1988.

Al momento de asumir el cargo de ministro, una gran proporción tenía edades entre 51 y 60 (43\%) y 41 y 50 años (38\%); el ministro más joven asumió el cargo a los 39 años (primeras gestiones de Javier Arias Stella, Armando Montes de Peralta y Paul Caro Gamarra) y la persona de mayor edad asumió el cargo de ministro a los 76 años (Óscar Ugarte Ubilluz). La mayoría de los ministros fueron médicos de profesión (73\%) seguidos de militares (17\%). Cerca de la mitad tenían especialidad médica y posgrado, y trabajan en áreas clínicas. El $41 \%$ tuvo participación política. Los cambios ministeriales ocurrieron con mayor frecuencia en los meses de julio (21\%), octubre (11\%), septiembre (10\%) y noviembre (10\%).

En todo el período analizado hubo 74 gestiones ministeriales. Ocho ministros tuvieron dos gestiones, Armando Montes de Peralta (1935-1936 y 1954-1955), Alberto Hurtado
(1947 y 1948), Alberto López (1948-1950 y 1955-1956), Javier Arias Stella (1963-1965 y 1967-1968), el Gral. Fernando MiróQuesada (1971-1975 y 1975), David Tejada (1985-1987 y 1989), Abel Salinas (2018 y 2020) y Óscar Ugarte (2008-2011 y 2021). Una ministra tuvo tres gestiones, Pilar Mazzetti (20042006, 2020 y 2021). El Gral. Miró Quesada fue ministro de dos presidentes del gobierno militar de 1968 a 1980.

La mediana de duración de la gestión de los ministros de Salud del Perú fue de 11 meses. El período de gestión ministerial más largo fue de 67 meses (Constantino Carvallo Alzamora fue ministro de Salud durante todo el primer gobierno de Manuel Prado), y los periodos más breves fueron de un día (al día siguiente de la designación del Javier Correa Miller se produjo un golpe de estado) y cuatro días (en la semana de la designación Abel Salinas se presentó la renuncia del presidente en funciones) (Tabla 2 y Tabla 3 ).

Se identificaron 46 eventos claves de la salud pública a lo largo de estos años clasificados en tres áreas, estrategia, impacto y regulación (Tabla 4).

Tabla 2. Características de las gestiones ministeriales de salud del Perú por periodo presidencial entre 1935 y 2021.

\begin{tabular}{|c|c|c|c|c|c|}
\hline \multirow{2}{*}{ Presidente } & \multirow[t]{2}{*}{$\begin{array}{l}\text { Número de gestiones } \\
\text { ministeriales }\end{array}$} & \multirow{2}{*}{$\begin{array}{l}\text { Número de } \\
\text { ministros }^{\mathrm{a}}\end{array}$} & \multirow[t]{2}{*}{ Periodo } & \multicolumn{2}{|c|}{$\begin{array}{c}\text { Duración de la gestión ministerial } \\
\text { de salud (meses) }\end{array}$} \\
\hline & & & & Media & Mediana \\
\hline Óscar Benavides Larrea & 5 & 5 & 1935-1939 & 10 & 6 \\
\hline Manuel Prado y Ugarteche & 1 & 1 & $1939-1945$ & 67 & 67 \\
\hline José Luis Bustamante y Rivero & 6 & 5 & $1945-1948$ & 7 & 7 \\
\hline Manuel Odría Amoretti & 6 & 5 & 1948-1956 & 15 & 16,5 \\
\hline Manuel Prado y Ugarteche & 5 & 5 & $1957-1962$ & 14 & 13 \\
\hline Junta Militar de Gobierno & 1 & 1 & $1962-1963$ & 12 & 12 \\
\hline Fernando Belaunde Terry & 4 & 3 & 1963-1968 & 15 & 17.5 \\
\hline Juan Velasco Alvarado & 3 & 3 & $1968-1975$ & 27 & 15 \\
\hline $\begin{array}{l}\text { Francisco Morales Bermúdez } \\
\text { Cerrutti }\end{array}$ & 5 & 5 & 1975-1980 & 11 & 11 \\
\hline Fernando Belaunde Terry & 3 & 3 & $1980-1985$ & 19 & 19 \\
\hline Alan García Pérez & 5 & 4 & $1985-1990$ & 13 & 11 \\
\hline Alberto Fujimori & 8 & 8 & $1990-2000$ & 15 & 15 \\
\hline Valentín Paniagua Corazao & 1 & 1 & $2000-2001$ & 8 & 8 \\
\hline Alejandro Toledo Manrique & 4 & 4 & 2001-2006 & 15 & 12 \\
\hline Alan García Pérez & 3 & 3 & 2006-2011 & 19 & 16 \\
\hline Ollanta Humala Tasso & 3 & 3 & 2011-2016 & 19 & 20 \\
\hline Pedro Pablo Kuczynski Godard & 3 & 3 & 2016-2018 & 6 & 3 \\
\hline Martin Vizcarra Cornejo & 5 & 5 & 2018-2020 & 6 & 4 \\
\hline Manuel Merino de Lama & 1 & 1 & 2020 & 0 & 0 \\
\hline Francisco Sagasti Hochhausler & 2 & 2 & $2020-2021$ & 2 & 2 \\
\hline Total & 74 & 70 & $1935-2021$ & 13,7 & 11 \\
\hline
\end{tabular}

${ }^{a}$ Los Drs. Montes, Ugarte, Salinas y Mazzeti fueron ministros de salud en diferentes gobiernos, el Gral. Miró-Quesada fue ministro de dos presidentes consecutivos y ocho ministros tuvieron dos gestiones ministeriales y un ministro tres gestiones ministeriales. En total hubo 64 personas que ocuparon el cargo de ministro de Salud. 


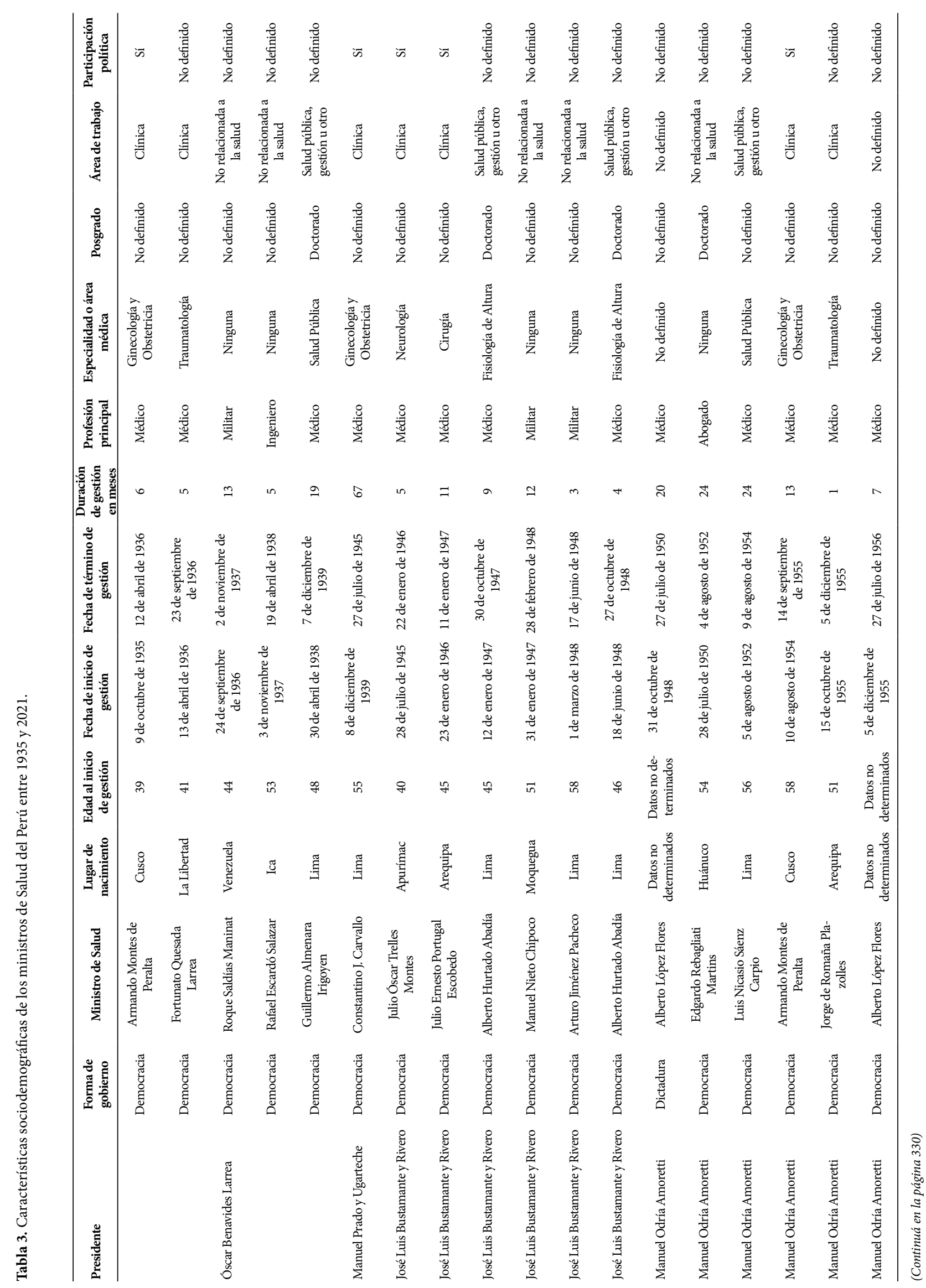




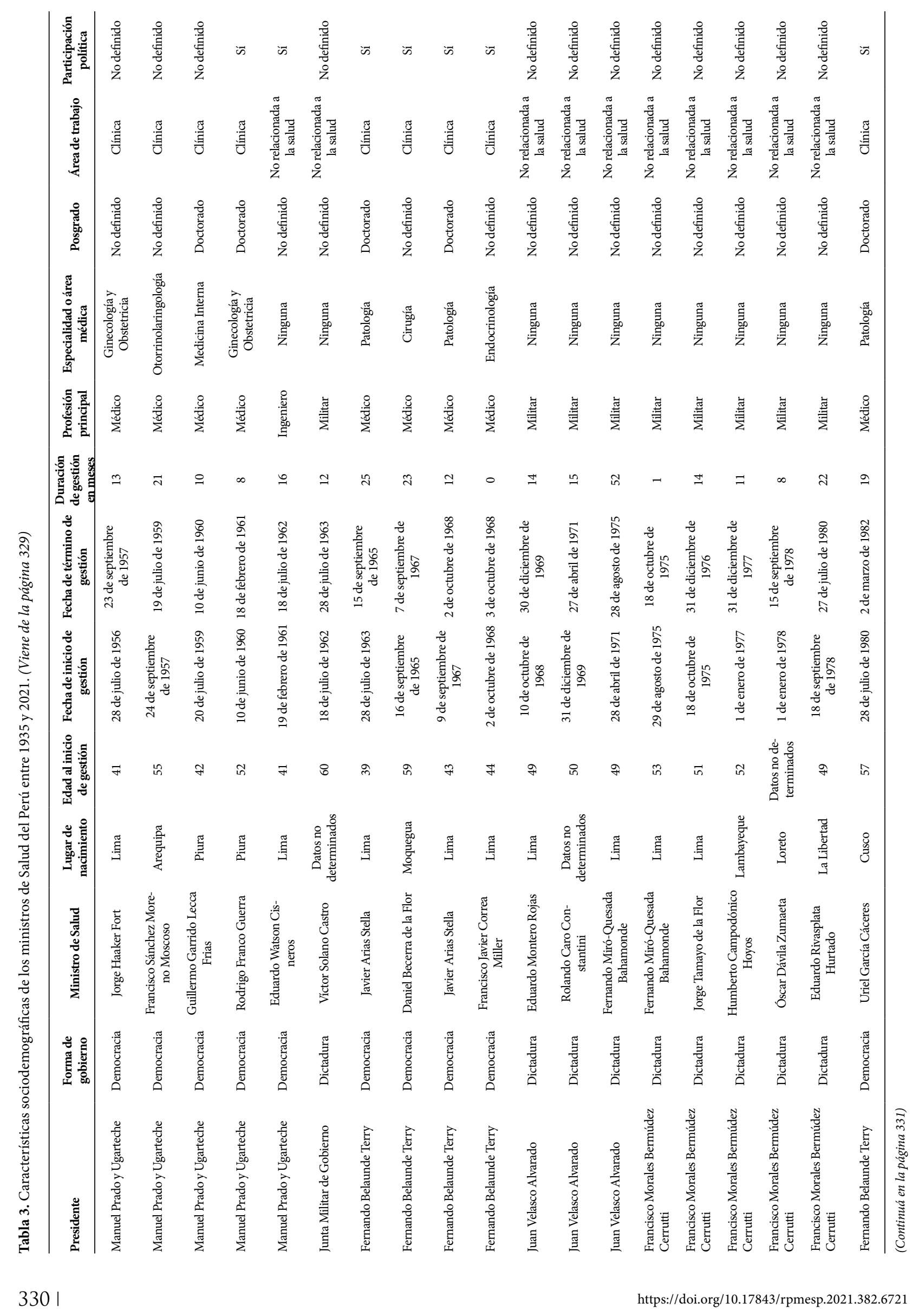




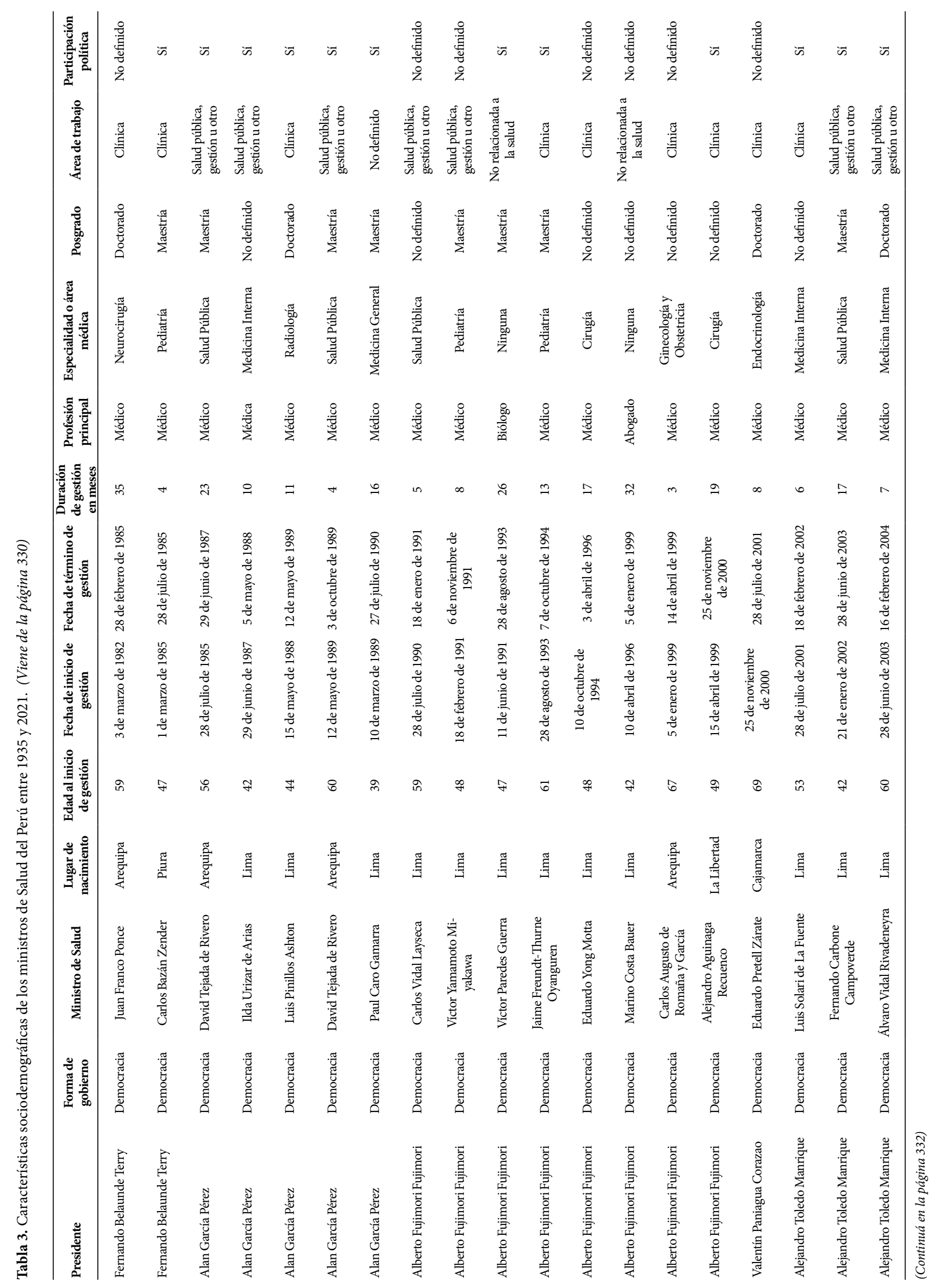




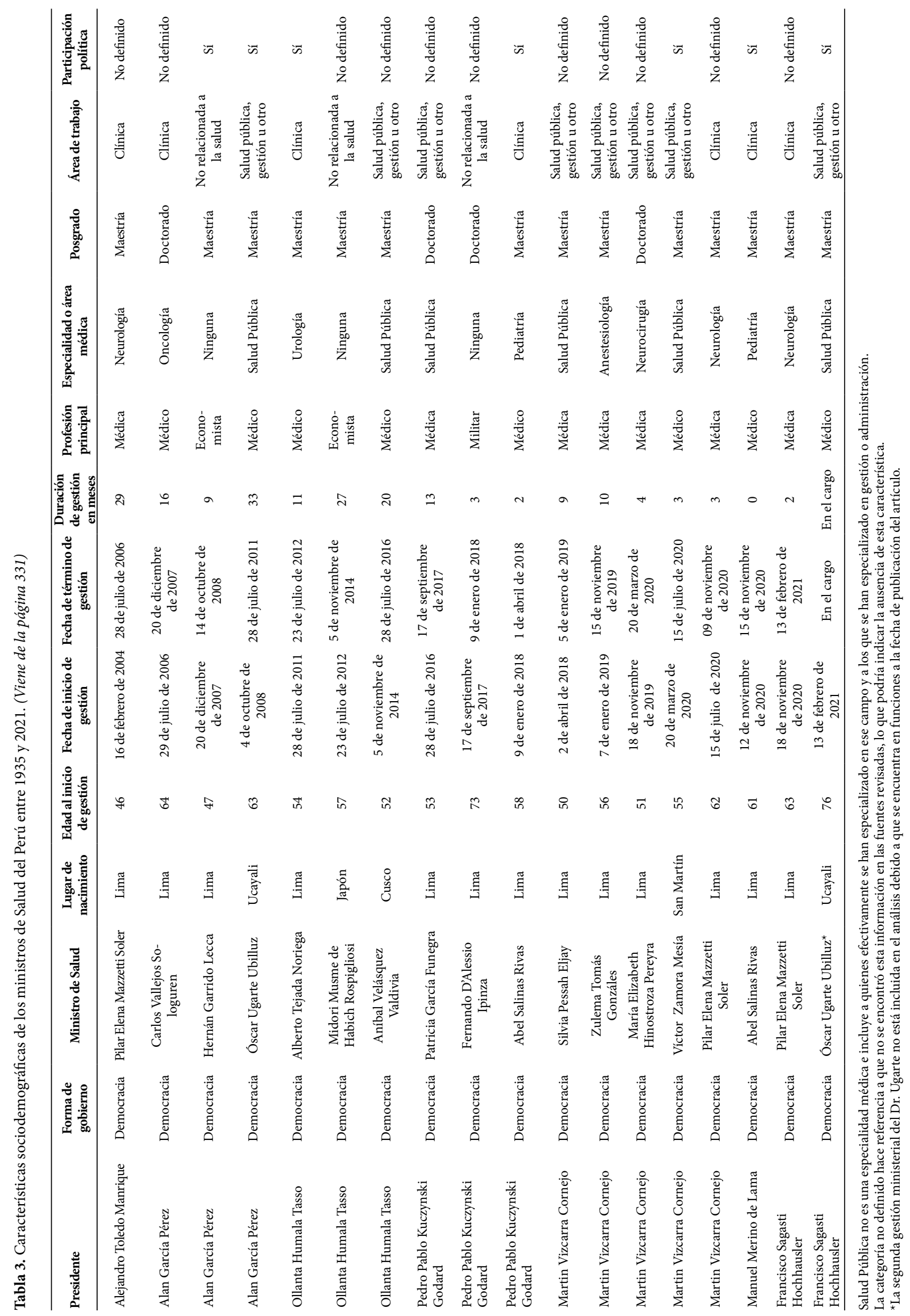


Tabla 4. Hitos de la salud pública en el Perú desde años próximos a la creación del Ministerio de Salud.

\begin{tabular}{|c|c|c|c|}
\hline Número & Año/periodo & Hito & Área \\
\hline 1 & 1933 & $\begin{array}{l}\text { Inclusión en la Constitución Política del rol del estado en la promoción física, moral y social; el reco- } \\
\text { nocimiento de los derechos individuales y sociales; y el impulso de legislación sobre salud e higiene. }\end{array}$ & Regulación \\
\hline 2 & 1933 & $\begin{array}{l}\text { Impulso de la promoción de la salud a través de las brigadas sanitarias indígenas («los rijcharis»o } \\
\text { «despertadores») en Puno. }\end{array}$ & Estrategia \\
\hline 3 & 1935 & $\begin{array}{l}\text { Creación del Ministerio de Salud del Perú denominado «Ministerio de Salud Pública, Trabajo y } \\
\text { Previsión Social». }\end{array}$ & Regulación \\
\hline 4 & 1936 & $\begin{array}{l}\text { Creación del Instituto Nacional de Higiene y Salud Pública de la integración de los laboratorios del } \\
\text { Instituto Nacional de Vacuna y Seroterapia, del laboratorio de Vacuna Antituberculosa del Dispen- } \\
\text { sario Antituberculoso, del Servicio Nacional Antipestoso y del Instituto Bacteriológico Peruano S.A. }\end{array}$ & Estrategia \\
\hline 5 & 1937 & Lanzamiento de la campaña antileprosa y creación del «Leprosorio de Huambo» en Andahuaylas. & Estrategia \\
\hline 6 & $1936-1948$ & $\begin{array}{l}\text { Inicio de la seguridad social con la ley del Seguro Social Obrero Obligatorio y la creación de la Caja } \\
\text { Nacional del Seguro Social; y posterior establecimiento del Seguro Social del Empleado, la Caja } \\
\text { Nacional del Seguro Social del Empleado y la creación de los Hospitales del Obrero y Empleado. }\end{array}$ & Regulación \\
\hline 7 & 1939 & Creación del Instituto Nacional del Cáncer. & Estrategia \\
\hline 8 & 1940 & $\begin{array}{l}\text { Oficialización del Instituto de Biología y Patología Andina como Instituto Nacional de Biología An- } \\
\text { dina. }\end{array}$ & Estrategia \\
\hline 9 & $1940-1941$ & $\begin{array}{l}\text { Creación del Departamento de Asistencia Hospitalaria y Beneficencia en el Ministerio de Salud para } \\
\text { impulsar el desarrollo de la infraestructura física de salud. }\end{array}$ & Estrategia \\
\hline 10 & $\begin{array}{l}1951 \\
1963-1964\end{array}$ & $\begin{array}{l}\text { Creación del Fondo Nacional de Salud y Bienestar Social en 1951, y elaboración del «Plan Bienal de } \\
\text { Obras Públicas» y «Plan de Construcciones Hospitalarias» entre 1963-1964 para ampliar la infraes- } \\
\text { tructura física en salud. Asimismo, la incorporación progresiva de hospitales de beneficencia pública } \\
\text { al Ministerio de Salud desde 1963. }\end{array}$ & Estrategia \\
\hline 11 & 1950-1966 & $\begin{array}{l}\text { Producción de la vacuna liofilizada contra la viruela y desarrollo de la estrategia de eliminación en } \\
\text { el Perú alcanzada en 1966. Producción local de vacunas para el sarampión, tos convulsiva, tétanos } \\
\text { y rabia. Exportación de vacunas a otros países. Producción del suero contra el veneno de la araña } \\
\text { domestica Loxoceles. }\end{array}$ & $\begin{array}{l}\text { Estrategia } \\
\text { Impacto }\end{array}$ \\
\hline 12 & $1957-1969$ & $\begin{array}{l}\text { Creación del Servicio Nacional de Erradicación de la Malaria (SNEM) con la reducción de la malaria } \\
\text { hasta un } 86 \% \text {. }\end{array}$ & Estrategia \\
\hline 13 & $1962-1964$ & $\begin{array}{l}\text { Creación del Servicio Especial de Salud Pública, el Centro de Capacitación de Salud y la Escuela } \\
\text { de Salud Pública del Perú orientados al desarrollo de la formación de recursos humanos en salud } \\
\text { pública y administración. }\end{array}$ & Estrategia \\
\hline 14 & 1969 & $\begin{array}{l}\text { Publicación del Código Sanitario y la Ley Orgánica del Sector Salud estableciendo el marco legal } \\
\text { sobre el derecho a la salud y la reorganización del Ministerio de Salud. }\end{array}$ & Regulación \\
\hline 15 & 1972 & Implementación del Programa Servicios Médico Rural Voluntario. & Estrategia \\
\hline 16 & 1973 & $\begin{array}{l}\text { Creación del Seguro Social del Perú de la unión de la Caja Nacional de Seguro Social, el Seguro } \\
\text { Social del Empleado y la Caja Nacional. }\end{array}$ & Regulación \\
\hline 17 & 1978 & $\begin{array}{l}\text { Participación en la organización de la Conferencia Internacional sobre Atención Primaria de Salud } \\
\text { de Almá-Atá. }\end{array}$ & Regulación \\
\hline 18 & 1978 & Creación del Sistema Nacional de Servicios de Salud (SNSS). & Regulación \\
\hline 19 & 1979 & $\begin{array}{l}\text { Inclusión en la Constitución Política del reconocimiento de la salud como un derecho de todos } \\
\text { incluyendo el derecho a la seguridad social y un acceso progresivo; y del requerimiento de la or- } \\
\text { ganización de un sistema nacional descentralizado y desconcentrado, que planifique y coordine la } \\
\text { atención integral de la salud. }\end{array}$ & Regulación \\
\hline 20 & 1980 & Creación del Instituto Peruano de Seguridad Social. & Regulación \\
\hline 21 & 1981-1982 & $\begin{array}{l}\text { Impulso de la atención primaria en salud con la promoción del uso de las sales de rehidratación oral } \\
\text { y medicamentos genéricos. }\end{array}$ & Estrategia \\
\hline 22 & 1983-1995 & $\begin{array}{l}\text { Creación de la Oficina de Bocio Endémico denominada posteriormente Programa Nacional de } \\
\text { Erradicación de los desórdenes por deficiencia de Iodo (DDI) y la eliminación del Bocio en el Perú } \\
\text { alcanzado en 1995. En } 1998 \text { la OPS/OMS-UNICEF-ICCIDD (International Council for Control of } \\
\text { Iodine Deficiency Disorders) certifica la eliminación virtual sostenida de los DDI. }\end{array}$ & Impacto \\
\hline 23 & $1985-1991$ & $\begin{array}{l}\text { Inicio de la campaña nacional de eliminación de polio en el Perú (VAN) y reporte del último caso } \\
\text { en } 1991 .\end{array}$ & Impacto \\
\hline 24 & 1985 & $\begin{array}{l}\text { Desarrollo de programas verticales de salud del MINSA relacionados a la nutrición, planificación } \\
\text { familiar e información y supervivencia infantil y promulgación de la Política Nacional de Población. }\end{array}$ & Estrategia \\
\hline 25 & 1989 & Inicio del Programa de Especialización en Epidemiología de Campo-PREC. & Estrategia \\
\hline 26 & $1990-1991$ & Creación del Instituto Nacional de Medicina Tradiciona.l & Estrategia \\
\hline 27 & 1991-1992 & $\begin{array}{l}\text { Organización y respuesta exitosa para el control de la epidemia del cólera con la promoción de sales } \\
\text { de rehidratación oral, la organización comunitaria «Motivadores de la comunidad», y el impulso de } \\
\text { las unidades de rehidratación oral (UROS comunales). }\end{array}$ & $\begin{array}{l}\text { Estrategia } \\
\text { Impacto }\end{array}$ \\
\hline 28 & 1992 & Creación del Instituto Nacional del Corazón del Seguro Social (INCOR). & Estrategia \\
\hline
\end{tabular}


Tabla 4. Hitos de la salud pública en el Perú desde años próximos a la creación del Ministerio de Salud (Viene de la página 333)

\begin{tabular}{|c|c|c|c|}
\hline Número & Año/Periodo & Hito & Área \\
\hline 29 & $1994-2020$ & $\begin{array}{l}\text { Implementación del Programa Salud Básica para Todos (PSBT) para ampliar la cobertura de salud } \\
\text { de la población en extrema pobreza y creación del Programa de Administración Compartida (PAC) } \\
\text { que originaron los Comités Locales de Administración en Salud CLAS que posteriormente adqui- } \\
\text { rieron rango de ley. }\end{array}$ & Estrategia \\
\hline 30 & 1994-1999 & $\begin{array}{l}\text { Inicio del Programa de Eliminación de Sarampión en el Perú con el logro de 95\% de cobertura na- } \\
\text { cional como meta global en el } 1999 .\end{array}$ & Impacto \\
\hline 31 & 1995 & $\begin{array}{l}\text { Creación del Programa de Control de ETS y SIDA (PROCETSS) para la prevención y cuidado } \\
\text { del SIDA y las ETS, reconocido como modelo de prevención en la región andina. }\end{array}$ & Estrategia \\
\hline 32 & 1997 & $\begin{array}{l}\text { Reforma de la seguridad social en salud con la reforma del Instituto Peruano de Seguridad Social a } \\
\text { través de la Ley de Modernización de la Seguridad Social en Salud. }\end{array}$ & Regulación \\
\hline 33 & 1997 & $\begin{array}{l}\text { Promulgación de la Ley General de Salud que establece el marco regulatorio esencial en temas sani- } \\
\text { tarios y de protección de la salud. }\end{array}$ & Regulación \\
\hline 34 & $1997-2002$ & $\begin{array}{l}\text { Financiamiento público para aumentar la cobertura de atención en salud e implementación del Se- } \\
\text { guro Escolar Gratuito (SEG), el Seguro Materno Infantil (SMI) y el Seguro Integral de Salud (SIS); el } \\
\text { SIS fue el resultado de un proceso de integración iniciado el } 2001 \text { del SEG y del SMI. }\end{array}$ & Regulación \\
\hline 35 & $1992-2001$ & $\begin{array}{l}\text { Diseño e implementación de los primeros proyectos de inversión en salud financiados por organis- } \\
\text { mos de cooperación financiera multilateral, así como un número importante de proyectos técnicos } \\
\text { y financieros con organismos de cooperación bilateral (Programa de Fortalecimiento de Servicios } \\
\text { de Salud, Proyecto de Salud y Nutrición Básica, Proyecto 2000, Proyecto Generación de Capacida- } \\
\text { des, entre otros). }\end{array}$ & Estrategia \\
\hline 36 & 2001 & $\begin{array}{l}\text { Creación del Sistema Integrado de Medicamentos (SISMED) para la compra centralizada de medi- } \\
\text { camentos. }\end{array}$ & Estrategia \\
\hline 37 & 2002 & Creación del Sistema Nacional Coordinado y Descentralizado de Salud (SNCDS). & Estrategia \\
\hline 38 & 2002 & $\begin{array}{l}\text { Creación del Acuerdo Nacional y la formulación como política de estado el Acceso Universal a los } \\
\text { Servicios de Salud y a la Seguridad Social. }\end{array}$ & Estrategia \\
\hline 39 & 2007 & Implementación de la Estrategia Nacional CRECER contra la desnutrición crónica infantil. & Estrategia \\
\hline 40 & 2009-2013 & $\begin{array}{l}\text { Impulso del aseguramiento universal en salud, creación de la Superintendencia Nacional de Asegu- } \\
\text { ramiento en Salud (SUNASA) y creación de la Superintendencia Nacional de Salud (SUSALUD). }\end{array}$ & Regulación \\
\hline 41 & 2012 & $\begin{array}{l}\text { Lanzamiento del Plan Nacional para la Atención Integral del Cáncer y el Mejoramiento del Acceso a } \\
\text { los Servicios Oncológicos en el Perú (Plan Esperanza). }\end{array}$ & Impacto \\
\hline 42 & 2013 & Impulso al marco de la reforma de salud con la promulgación de 23 Decretos Legislativos. & Regulación \\
\hline 43 & 2013-2018 & $\begin{array}{l}\text { Promulgación de la ley y reglamento de «Promoción de Alimentación Saludable para Niños, Niñas } \\
\text { y Adolescentes» y el «Manual de Advertencias de Alimentos Procesados» con el uso de octógonos . }\end{array}$ & Regulación \\
\hline 44 & $2003-2015$ & $\begin{array}{l}\text { Implementación de vacuna de sarampión, rubéola y paperas en 2003, e inicio de Campaña Nacional } \\
\text { para la eliminación de la Rubéola y el Síndrome de Rubéola Congénita (2006), y certificación de la } \\
\text { eliminación de la rubéola en } 2015 \text {. }\end{array}$ & Impacto \\
\hline 45 & 2016 & $\begin{array}{l}\text { Promulgación de la Ley que fortalece la autoridad sanitaria nacional y recupera su capacidad de in- } \\
\text { tervención mediante el Centro Nacional de Epidemiología, Prevención y Control de Enfermedades } \\
\text { (CDC). }\end{array}$ & $\begin{array}{l}\text { Regulación } \\
\text { Estrategia }\end{array}$ \\
\hline 46 & 2019 & Establecimiento de medidas para acelerar la Cobertura Universal de Salud (CUS). & Regulación \\
\hline
\end{tabular}

\section{DISCUSIÓN}

En el Perú, la duración de la gestión ministerial en salud desde su creación en 1935 ha sido variable con una mediana de duración de 11 meses y una media de 13,7 meses.

La primera ministra de salud fue designada, aproximadamente 52 años después de la creación de esta entidad y 31 años desde la promulgación del derecho al voto femenino. Conforme avanza el siglo XXI la presencia de la mujer en el cargo de ministra de Estado ha sido cada vez más frecuente.

En comparación con otros países, la duración de la gestión ministerial en el Perú es breve y se ha reducido en años recientes. Un estudio que revisó la duración de la gestión de los ministros de salud de 23 países de 1990 al 2010, reportó una mediana de 20 meses, siendo Suiza (96 meses), Estados Unidos (47,5 meses) y Singapur (42 meses) los países con las medianas de duración más extensas y Corea del Sur (10 meses), Polonia y República Checa (13 meses) los países con medianas de duración más breves ${ }^{(17)}$. Esta revisión incluyó dos países latinoamericanos, Chile (32 meses) y Brasil (13 meses). Asimismo, el estudio reportó una correlación estadísticamente significativa de orden negativo entre el número de ministros y cuatro indicadores generales (el Îndice de Desarrollo Humano (IDH), el Producto Bruto Interno per cápita, el acceso a agua potable y el acceso a la salud). Así, se pudo apreciar que países con altos índices de desarrollo humano y economías avanzadas tienen los promedios de duración más altos.

Un estudio en Estados Unidos ha reportado periodos largos de duración en cargos de funcionarios de salud. En 
un periodo de más de 35 años se encontró que el promedio de duración en el cargo de altos funcionarios de salud fue de 63 meses; aunque se señala que la duración en el cargo ha venido disminuyendo con los años y que es menor en el sector público en comparación al privado ${ }^{(18)}$. En este estudio se encontró una relación positiva entre la duración en un cargo con la posición que ocupa un Estado de este país en indicadores de salud. Algunos funcionarios encuestados que habían dejado sus cargos señalaron que su periodo fue breve y que su salida tuvo un impacto negativo en el funcionamiento de su institución ${ }^{(18)}$. En otro estudio relacionado al primero, se describió que aquellos cargos elegidos por consejos directivos tuvieron una mayor duración que aquellos elegidos por autoridades políticas u otros directivos ${ }^{(19)}$.

Fundamentos teóricos han apoyado los planteamientos sobre los impactos negativos directos que tendría la rotación frecuente de altos ejecutivos en la gestión de una institución del sector privado o público. La teoría de los escalafones superiores desarrollada por Hambrick y Mason propone por ejemplo que el conocimiento, los valores y las percepciones de un equipo de alta dirección están relacionados con las decisiones que toman y a su vez con su efectividad en una organización. A partir de esta propuesta se planteó un modelo de cinco etapas que resume los diferentes momentos en el periodo de gestión de altos ejecutivos (ciclo de vida de la gestión), principalmente para el sector privado pero aplicable además al sector público y a la gestión de gobierno. $\mathrm{La}$ «respuesta al mandato, la experimentación, la selección de una narrativa, la convergencia y la disfunción» son las distintas etapas interrelacionadas de este modelo. En el contexto de este enfoque, un periodo corto de gestión de un directivo no sería suficiente para recorrer cada una de las cinco etapas y en consecuencia los resultados de su gestión podrían ser significativamente afectados ${ }^{(20,21)}$.

El tiempo de permanencia en una gestión puede obedecer a la crisis del gabinete, fin del periodo legislativo, errores individuales, presión de grupos de poder, términos del mandato presidencial, entre otros ${ }^{(22-24)}$. En Chile, un análisis estadístico de los factores relacionados a la rotación de los 180 ministros designados entre 1990 y 2010, encontró que la duración ministerial estuvo asociada a dos eventos críticos, las crisis económicas y los escándalos de corrupción ${ }^{(24)}$. Se ha señalado que la diferencia entre sectores, la débil capacidad institucional, la indecisión política y los intereses creados hacen compleja y difícil una gestión y el ejercicio del cargo ministerial ${ }^{(25)}$.

En el caso de altos directivos del sector público en salud, como el de ministro, puede ocurrir algo similar a lo que se describe para el sector privado. Los periodos breves de gestión pueden tener un impacto en el aprendizaje y habilidad política lo que se reflejaría en la capacidad y efectividad en la gestión. El cambio de ministro implica cambios de dirección y nuevas visiones con un deseo por imprimir un legado par- ticular que significan un enorme riesgo en la sostenibilidad de políticas de Estado a largo plazo. Es usual que las políticas vuelvan a foja cero. Los equipos cambian, debido a la necesidad de contar con un personal de confianza; no obstante, como ocurre al más alto nivel, y en una especie de cascada, los cambios de equipos ocurren en varios niveles incluso los más técnicos.

Una rotación alta en el Perú podría dificultar la implementación de políticas de Estado en salud. Probablemente esta situación particular se relaciona con la inestabilidad de la propia democracia a lo largo de nuestra historia republicana. Una muestra de esta inestabilidad se expresa en lo ocurrido solo en los últimos cinco años en que se ha tenido tres presidentes de la República y once gestiones ministeriales en salud, seis de las cuales se han producido desde el inicio de la pandemia de la COVID-19, tres de ellas con ministros que tenían por lo menos una gestión anterior. La adecuada capacidad técnica, liderazgo y respaldo político podría contribuir a dar estabilidad a la gestión de los ministros y al fortalecimiento institucional, y por lo tanto al cumplimiento del rol de la autoridad sanitaria en la cambiante y globalizada realidad de la sociedad actual, siempre y cuando existan políticas de Estado en salud claramente definidas y consensuadas.

Alolargo dela historia ministerial de salud, sehan identificado una serie de hitos que han marcado avances significativos en la mejora de la salud, el bienestar y la vida de todos los peruanos. Una de las características de estos hitos es que la mayoría de los hechos claves, simples o complejos, se han logrado a lo largo de varios periodos ministeriales; por tanto no son eventos transversales pese a su ubicación en fechas determinadas, sino longitudinales producto de procesos continuos y evolutivos. Otra es que son producto del concurso de varios actores, incluso de personas u organizaciones, independientes de las gestiones ministeriales. Entre los logros se destacan el desarrollo de estrategias y programas para prevenir y proteger a las personas y minimizar las amenazas a la salud pública. Otros logros incluyen el desarrollo de políticas, reformas e infraestructura sanitaria. Estas características resaltan la importancia de dar continuidad y sostenibilidad explícita a las políticas públicas en el tiempo para lograr impacto; en la medida que se trata de procesos complejos, requieren de acuerdos implícitos o explícitos y de la coparticipación de diferentes actores inclusive por fuera del sector salud. Esta lista no es exhaustiva y no delimita los hitos por gestión ministerial o refleja la efectividad de las mismas, en parte por lo breve de las gestiones y en parte porque los resultados se han obtenido después de varios periodos y son difíciles de aislar, no obstante, reflejan el impulso de los liderazgos que iniciaron o continuaron con los procesos. En la lista no se describe los detalles de los procesos y no se realiza un análisis de los efectos específicos o del contexto en los que ocurrieron. Tampoco se describen los posibles retrocesos en estos periodos. Otra de las limitaciones es que el proceso de identificación no 
fue sistemático y por tanto puede ser subjetivo. Sin embargo, está basado en fuentes históricas y en nuestro conocimiento es la primera lista orientada a resumir los principales logros en salud pública desde la creación del ministerio a la actualidad.

En conclusión, la duración de la gestión ministerial en el Perú tiene un amplio rango de variación y es breve comparada con la de otros países, especialmente durante el último quinquenio. Se recomienda considerar en las transiciones ministeriales y de gobierno nacional, los posibles efectos negativos de las gestiones muy breves sobre el sostenimiento de las políticas de Estado en salud y los mecanismos para minimizarlos. Por otro lado, es necesario estudiar las causas de la breve duración de las gestiones ministeriales, así como sus consecuencias, tanto sobre el desarrollo institucional como sobre la implementación, continuidad e impacto de las políticas de Estado. De igual forma, realizar estudios complementarios sobre los criterios (y de ser posible las circunstancias) en la elección de un ministro en la cartera de salud y de la relación que estableció éste con la arquitectura del poder dentro del aparato público, así como la realización de análisis comparativos entre los períodos de duración y el impacto sobre los resultados en salud.

Contribución de autoría: EG y FC concibieron la idea y redactaron la versión inicial; VC, GH, AC, WQ y RE recolectaron la información; todos revisaron críticamente el manuscrito y aprobaron la versión final.

Financiamiento: Autofinanciado.

Conflictos de interés: Los autores declaran no tener conflictos de interés.

\section{REFERENCIAS BIBLIOGRÁFICAS}

1. Bustíos Romaní C. Cuatrocientos años de la salud pública en el Perú (1533-1933). 1ra ed. Lima: Fondo Editorial, Universidad Nacional Mayor de San Marcos : CONCYTEC, Consejo Nacional de Ciencia y Tecnología; 2004. 707 p. (Serie Coediciones).

2. Espinosa L, Antonio J. El centenario del primer Ministerio de Salud del mundo. ACIMED. 2019; 19(1).

3. Cuesta JLL de la, Frenk J. Pluralismo estructurado: hacia un modelo innovador para la reforma de los sistemas de salud en América Latina. Washington DC: Banco Interamericano de Desarrollo; 1997. 40 p.

4. Gozzer E. Hacia la Cobertura Universal en Salud. Primera. Lima: Ministerio de Salud del Perú; 2015. 248 p.

5. Evans P, Rauch JE. Bureaucracy and Growth: A Cross-National Analysis of the Effects of "Weberian" State Structures on Economic Growth. Am Sociol Rev. 1999;64(5):748-65. doi: 10.2307/2657374.

6. Henderson J, Hulme D, Jalilian H, Phillips R. Bureaucratic Effects: 'Weberian' State Agencies and Poverty Reduction. Sociology. 2007 Jun;41(3):515-32. doi: 10.1177/0038038507076620.

7. Dahlström C, Lapuente V, Teorell J. The Merit of Meritocratization: Politics, Bureaucracy, and the Institutional Deterrents of Corruption. Polit Res Q. 2012;65(3):656-68. doi: 10.1177/1065912911408109.

8. Ministerio de Salud del Perú. Reglamento de Organización y Funciones del Ministerio de Salud [Internet]. Decreto Supremo No 008-2017-SA 2017 p. 93. Disponible en: ftp://ftp2.minsa.gob.pe/normaslegales/2017/ rof_2017.pdf.

9. Bustíos Romaní C. La salud pública, la seguridad social y el Perú demoliberal (1933 - 1968). 2005. Lima: CONCYTEC, Consejo Nacional de Ciencia y Tecnología; 2005. 658p.

10. Bustios Romaní C, Chávez MM, Murillo JP. Servicio Cooperativo Interamericano de Salud Pública: una historia de 20 años de colaboración con el Perú. An Fac Med. 2019;80(4):515-27. doi: 10.15381/anales.v80i4.17254.

11. Salaverry García O, Delgado Matallana G, editors. Historia de la medicina peruana en el siglo XX. Tomos I y II. Lima: Univ. Nacional Mayor de San Marcos, Fondo Editorial; 2000. 1382 p.

12 Lazo O, Alcalde-Rabanal J, Espinosa-Henao O. El sistema de salud en Peru: situación y desafios. Lima, Perú: Colegio Médico del Perú, REP; 2016.

13. Cueto M. El regreso de las epidémias: salud y sociedad en el Perú del siglo XX. 1ra ed. Lima, Perú: Instituto de Estudios Peruanos; 1997. 256 p. (Serie Estudios históricos).

14. Álvarez Carrasco R. Colegio Médico del Perú - Una visión retrospectiva de sus primeros 50 años de historia [Internet]. Lima, Perú:

Colegio Médico del Perú, REP; 2017 [citado el 6 de noviembre de 2020]. Disponible en: http://localhost:8080/xmlui/handle/CMP/57.

15. Organización Panamericana de la Salud. Cien años de cooperación al Perú: 1902-2002 [Internet]. Lima: OPS; 2002 [citado el 6 de noviembre de 2020]. Disponible en: https://iris.paho.org/handle/10665.2/41384.

16. Agencia de los Estados Unidos para el Desarrollo Internacional, USAID. Una historia de 70 años de colaboración en salud con el Perú [Internet]. 2a ed. Baltimore: Johns Hopkins University y Tarea Asociación Gráfica Educativa; 2015 [citado el 6 de noviembre de 2020]. Disponible en: https://www.usaid.gov/documents/1864/una-historia-de-70-a\%C3\%Blos-de-colaboraci\%C3\%B3n-en-salud-con-el-peru.

17. Ferraz MB, Azevedo RT. Ministers of Health: short-term tenure for long-term goals?. Sao Paulo Med J Rev Paul Med. 2011;129(2):77-84 doi: 10.1590/s1516-31802011000200005.

18. Menachemi N, Danielson EC, Tilson HA, Yeager VA, Sellers K, Halverson PK, et al. Tenure and Turnover Among State Health Officials From the SHO-CASE Survey: Correlates and Consequences of Changing Leadership. J Public Health Manag Pract JPHMP. 2020;26(1):23-31. doi: 10.1097/PHH.0000000000000991.

19. Halverson PK, Lumpkin JR, Yeager VA, Castrucci BC, Moffatt S, Tilson H. High Turnover Among State Health Officials/Public Health Directors: Implications for the Public's Health. J Public Health Manag Pract JPHMP. 2017;23(5):537-42. doi: 10.1097/PHH.0000000000000639.

20. Hambrick DC, Fukutomi GD. The seasons of a CEO's tenure. Acad Manag Rev Acad Manag. 1991;16(4):719-42.doi: 10.2307/258978.

21. Antonini-Bortoluzzi D, Jimenez-Franco MA, Aparecido-Silva S, João-Lunkes R. La influencia de los escalafones superiores en el retorno sobre el activo de las empresas del sector de consumo no cíclico. ADMinist. 2017;(31):59-74. doi: 10.17230/ad-minister.31.4.

22. Grossman E, François A. Who are the ministers of the Fifth Republic?. Fr Polit. 2013;11(3):272-83. doi: 10.1057/fp.2013.11.

23. Avendaño O, Dávila M. Rotación ministerial y estabilidad coalicional en Chile, 1990-2010. Política. 2012;50(2):87-108. doi: 10.5354/07161077.2013.25968.

24. González-Bustamante B, Olivares A. Cambios de gabinete y supervivencia de los ministros en Chile durante los gobiernos de la Concertación (19902010). Colomb Int. 2016;(87):81-108. doi: 10.7440/colombiaint87.2016.04.

25. Nishtar S. Reflections from my tenure as minister. Lancet Lond Engl. 2015;385(9971):910-3. doi: 10.1016/S0140-6736(14)61284-8. 